\title{
Valve-based flow focusing for drop formation
}

\section{Citation}

Abate, Adam R., Mark B. Romanowsky, Jeremy J. Agresti, and David A. Weitz. 2009. “ValveBased Flow Focusing for Drop Formation." Applied Physics Letters94 (2): 023503. https:// doi.org/10.1063/1.3067862.

\section{Permanent link}

http://nrs.harvard.edu/urn-3:HUL.InstRepos:41511268

\section{Terms of Use}

This article was downloaded from Harvard University's DASH repository, and is made available under the terms and conditions applicable to Other Posted Material, as set forth at http:// nrs.harvard.edu/urn-3:HUL.InstRepos:dash.current.terms-of-use\#LAA

\section{Share Your Story}

The Harvard community has made this article openly available.

Please share how this access benefits you. Submit a story.

\section{Accessibility}




\title{
Valve-based flow focusing for drop formation
}

\author{
Adam R. Abate, Mark B. Romanowsky, Jeremy J. Agresti, and David A. Weitz ${ }^{a)}$ \\ Department of Physics and School of Engineering and Applied Science, Harvard University, \\ Cambridge, Massachusetts 02139, USA
}

(Received 10 September 2008; accepted 6 December 2008; published online 14 January 2009)

\begin{abstract}
Microfluidic devices can produce highly monodisperse drops at kilohertz rates using flow-focus drop formation. We use single-layer membrane valves to control, in real time, the dimensions of the flow-focus drop makers. This allows drop size and frequency to be controlled in real time and without adjusting flow rates. (C) 2009 American Institute of Physics. [DOI: 10.1063/1.3067862]
\end{abstract}

Picoliter drops made in microfluidic devices can serve as individual compartments for chemical reactions ${ }^{1}$ and can be processed at kilohertz rates with high precision. ${ }^{2}$ This combination of speed and containment is very useful for highthroughput screening, for discovering novel drugs, ${ }^{3}$ for sorting analytes and worms, ${ }^{4}$ and for directed evolution of enzymes and cells. ${ }^{5}$ For applications in microfluidics, precision control of the drops is essential. In droplet based screening, drops must be formed, merged, and sorted in a particular sequence and with precise timing. This has stimulated the development of microfluidic systems that provide exceptional control of drops. ${ }^{6,7}$ Drop-on-demand microfluidics allows drop formation to be actively controlled by creating drops as needed. ${ }^{8}$ This allows operations between drops, such as drop merger, to be carefully timed and controlled. However, drop-on-demand devices are difficult to operate; they require sophisticated external devices, such as automated pumps, actuators, and computers, to carefully monitor and execute all operations. The intermittent nature of the devices also significantly limits their speed and their usefulness for high-throughput screens.

Alternatively, continuous-flow microfluidics can operate at very high throughput. ${ }^{2}$ As with drop-on-demand devices, continuous-flow microfluidics can be structured like an assembly line, enabling complex tasks to be efficiently performed. ${ }^{7}$ With soft lithography, devices can be reproducibly engineered and easily fabricated. ${ }^{9}$ This allows implementation of passive forms of control. For example, channel dimensions can be carefully selected to cause drops to flow as pairs in preparation for electrically induced merger. ${ }^{2,10}$ Drops can be steered based on size, ${ }^{11}$ slowed in large incubation chambers, ${ }^{7}$ and ordered into a linear array for individualized detection. ${ }^{12,13}$ However, continuous-flow microfluidics can be difficult to operate robustly. Coupled devices can display long-lived or chaotic oscillations in which flows vary uncontrollably over time and never reach a steady state. ${ }^{14}$ To increase control, sensors can monitor flows and automatically adjust pumps to compensate for variations. However, due to compliance of the pumps and devices, flow rate changes controlled at the pump can have a slow response time, lagging by seconds, or even minutes, on different parts of the device. ${ }^{15}$ For typical drop production frequencies of $1 \mathrm{kHz}$, this is a significant limitation. Moreover, changes in flow rate affect the performance of the device as a whole and cannot control individual components in a microfluidic circuit. An optimal system would combine the simplicity and

${ }^{a)}$ Electronic mail: weitz@seas.harvard.edu. throughput of continuous-flow microfluidics, with the timing and control of drop-on-demand devices.

In this paper, we introduce a simple system that provides localized, fast response-time control of drops in continuousflow microfluidics. We use single-layer membrane valves to adjust the local dimensions of the drop formation junction. We introduce two methods to control flow focusing with valves. First, we use membrane valves to control the dimensions of the drop formation orifice, thereby enabling drop size to be controlled. Second, we also use membrane valves to control the dimensions of the flow-focus side channels, thereby enabling drop frequency to be controlled. Both forms of control are localized at the flow-focus junction. Neither requires adjustment of the total flow rate. Together, they enable drop formation to be controlled in real time, over a wide range, and on a single, simple to fabricate microfluidic device.

The microfluidic devices are fabricated using singlelayer soft lithography in poly(dimethylsiloxane) (PDMS). We produce water drops in HFE-7500 fluorocarbon oil with $5 \%$ (vol/vol) $1 H, 1 H, 2 H, 2 H$-perfluoro-1-octanol (Sigma), stabilized by 1.8 wt $\%$ of the fluorosurfactant ammonium carboxylate of DuPont Krytox $157 .{ }^{16}$ For the valves we use single-layer membrane valves, which exist in the same plane as the microfluidic channels they control, thereby enabling the entire microfluidic device to be fabricated conveniently in a single-layer mold. ${ }^{17}$ For optimal valve performance, the PDMS membrane must be as thin and flexible as possible. ${ }^{17}$ To make thin membranes that can be reliably fabricated, we design the membrane width to be $20 \mu \mathrm{m}$; due to limited resolution of the fabrication process, they reliably turn out at approximately $13 \mu \mathrm{m}$ in width in the PDMS device. To make the membranes very flexible, we use a 12-to-1 PDMSto-cross-linker ratio and bake the devices at $65{ }^{\circ} \mathrm{C}$ for $1 \mathrm{~h}$. To actuate the valves, we pressurize the valve control channels. For the size control device, we pressurize the channels with a hand-held syringe filled with water. For the frequency control device, we use an electronically controlled pressure regulator (Parker Hannefin, VSO series); this allows highfrequency actuations to be applied to the valve, which we use to impose high-frequency signals onto the drop trains.

We introduce two forms of valve-based flow focusing. In the first, we use valves to control drop size. For a flow-focus drop maker in the dripping regime, the drop size is proportional to the width of the flow-focus orifice and inversely proportional to the continuous phase flow speed. This is evident in the approximation for a channel with circular cross section, $\eta_{c} u_{c} d_{d} \sim \gamma d_{o}$, where $\eta_{c}$ is the viscosity of the continuous phase, $u_{c}$ is the flow rate of the continuous phase, $d_{d}$ 

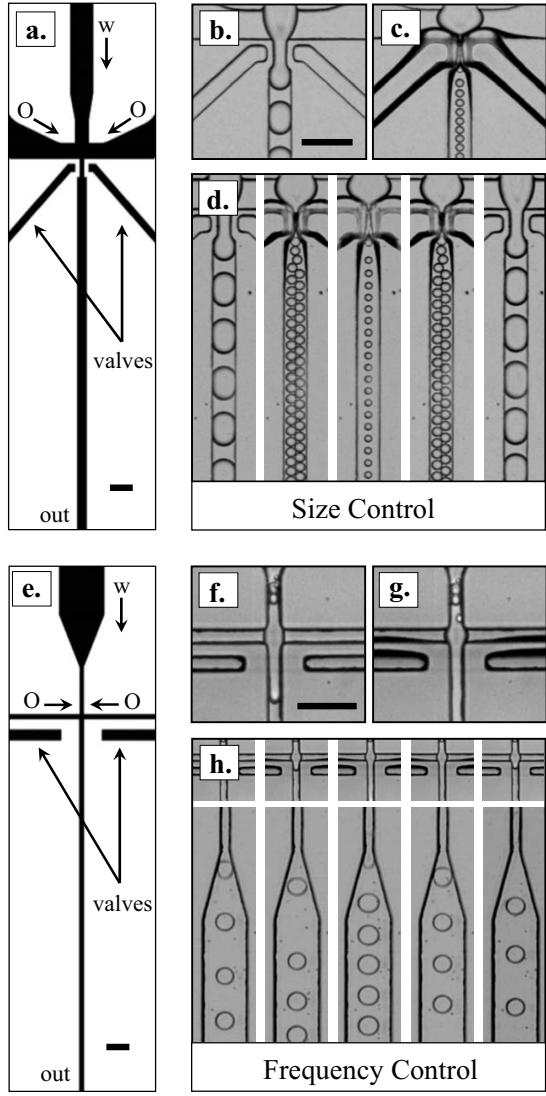

FIG. 1. (a) Schematic of size control drop maker; the valve control channels are indicated by the black arrows; the device is $50 \mu \mathrm{m}$ tall and the nozzle is $20 \mu \mathrm{m}$ in width. Photomicrographs of size control device with (b) valve unactuated so that large drops are produced, (c) valve actuated so that small drops are produced, and (d) over a cyclical range of valve actuations; flow rates are $200 \mu \mathrm{l} / \mathrm{h}$ for the dispersed and continuous phases. (e) Schematic of frequency control drop maker with valve edges indicated by black arrows; the device is $50 \mu \mathrm{m}$ tall and $20 \mu \mathrm{m}$ in width. Photomicrographs of frequency control devices with (f) valves unactuated so that drops are produced slowly, (g) valves actuated so that drops are produced more quickly, and (h) valves actuated over a cyclical range; flow rates are $500 \mu \mathrm{l} / \mathrm{h}$ for the dispersed phase and $2000 \mu \mathrm{l} / \mathrm{h}$ for the continuous phase. Scale bars for all figures denote $100 \mu \mathrm{m}$.

is the diameter of the drop produced, $\gamma$ is the surface tension, and $d_{o}$ is the diameter of the drop formation orifice. ${ }^{18} \mathrm{We}$ thus position valves on both sides of the drop formation orifice to control its width, as shown in the diagram in Fig. 1(a); the valve control channels are indicated by the black arrows in the diagram. (AUTOCAD design files of the devices, as well as movies of their operation, are available online). ${ }^{19} \mathrm{We}$ design the side channels of the flow-focus junction to be $60 \mu \mathrm{m}$ wide so that the vertical deflection of the membrane does not affect the continuous phase flow rate. The $20 \mu \mathrm{m}$ wide and $50 \mu \mathrm{m}$ tall orifice produces large drops when the valves are not actuated and small drops when the valves are actuated even at fixed volumetric flow rate. To measure drop size, we record high speed camera movies of the flow-focus junction (available online). ${ }^{19}$ From the images, we extract the drop diameter and orifice width using image analysis. When the valves are not actuated, the orifice is fully open so that large drops are produced, as shown in Fig. 1(b). By contrast, when the valve is actuated, the orifice width is reduced and the local flow velocity increased so that small drops are produced, as shown in Fig. 1(c); example images from a cyclic actuation are shown in Fig. 1(d). The size control device allows drop size to be controlled from 200 down to $7 \mu \mathrm{m}$ at

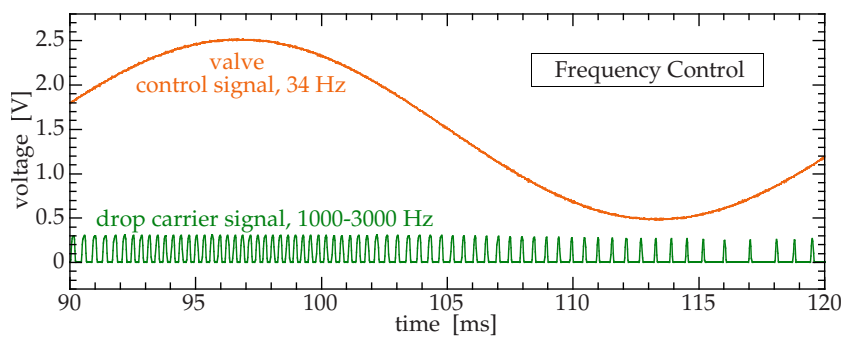

FIG. 2. (Color online) Raw time traces of the valve actuation signal (upper orange curve) and fluorescent drop signal (lower green curve with spikes); each spike corresponds to a drop flowing past the optical detector.

fixed flow rate of $200 \mu \mathrm{l} / \mathrm{h}$ for the dispersed and $200 \mu \mathrm{l} / \mathrm{h}$ for the continuous phases.

The frequency with which drops are produced depends on the flow speeds of the dispersed and continuous phases as they are injected into the flow-focus junction. ${ }^{14,20}$ To produce drops at a high frequency, we use a narrow drop formation orifice that is $10 \mu \mathrm{m}$ wide and $50 \mu \mathrm{m}$ tall, and high flow rates of $500 \mu \mathrm{l} / \mathrm{h}$ for the dispersed and $2000 \mu \mathrm{l} / \mathrm{h}$ for the continuous phases. To control drop frequency, we position valves on the side channels of the flow-focus junction, as shown in the diagram in Fig. 1(e); the edges of the valves are indicated by the black arrows; bypass channels allow excess fluid to circumvent the flow-focus junction. When the valves are not actuated, the continuous phase flow rate is at a maximum so that drops are produced slowly, as shown in Fig. 1(f). By contrast, when the valve is actuated, the continuous phase flow rate is reduced so that drops are produced at a higher volume fraction and more quickly, as shown in Fig. $1(\mathrm{~g})$; example images from a cyclic actuation are shown in Fig. 1(h). ${ }^{19}$ Actuating the valve thus allows the periodicity of the drops to be controlled while changing their size very little. To measure drop frequency, we flow fluorescein-filled drops past a $488 \mathrm{~nm}$ laser and collect the emitted fluorescence with a photomultiplier tube (PMT) behind a $532 \mathrm{~nm}$ band pass filter. An example fluorescent drop signal is plotted as a function of time in Fig. 2; each short spike represents a fluorescent drop that has moved past the optical axis of the
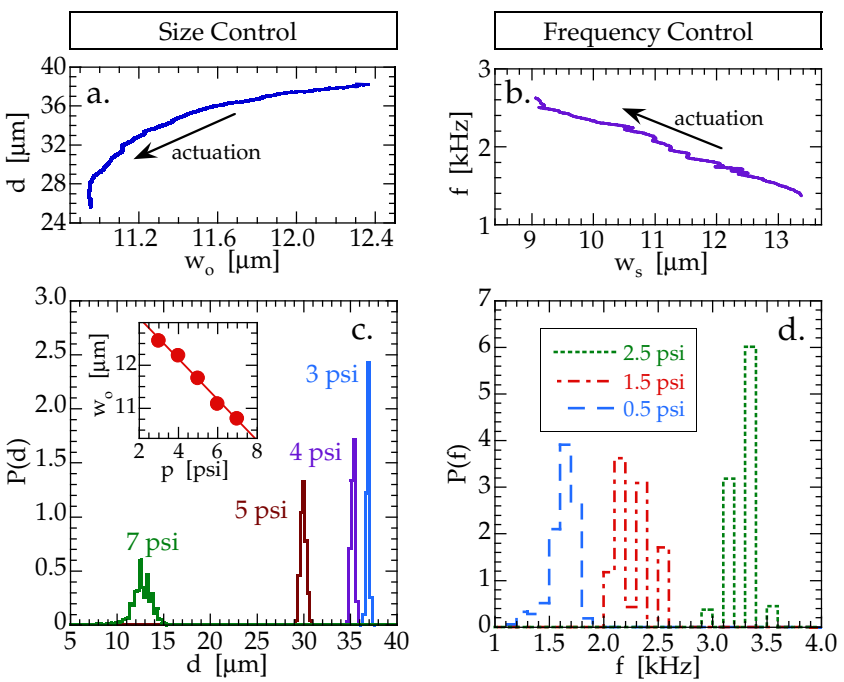

FIG. 3. (Color online) Response functions of (a) drop size $d$ as a function of orifice width $w_{o}$ and (b) drop production frequency $f$ as a function of side channel width $w_{s}$. (c) Drop size distributions for different valve actuations; the inset shows the optically measured width of the orifice as a function of the pressure applied to the valve. (d) Drop production frequency distributions for three valve actuations. 

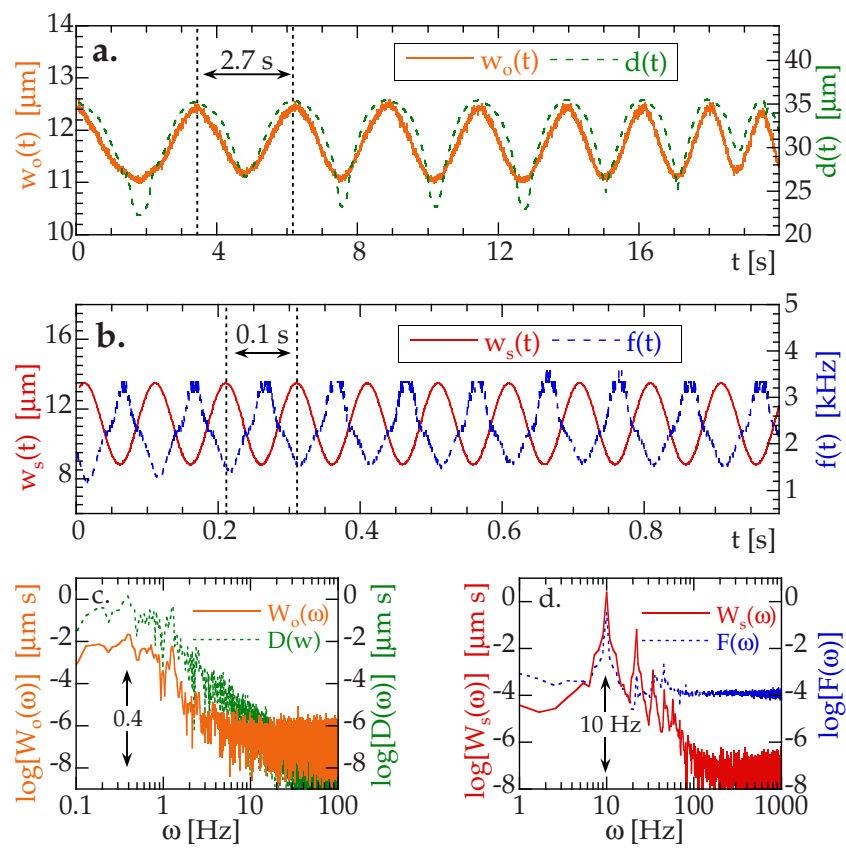

FIG. 4. (Color online) Time traces of (a) orifice width and drop diameter and (b) side channel width and drop frequency. Power spectra of time traces for (c) orifice width and drop diameter and (d) side channel width and drop frequency.

PMT. We also plot the valve control signal, which is the upper, slowly varying sinusoidal curve in the figure. The spacing of the spikes changes in response to the actuation of the valve because the drop frequency changes, as shown in Fig. 2.

Drop size and frequency can thus be controlled by actuating the flow-focus valves. The response of the drops depends on the amplitude and frequency of the valve actuation. To quantify this control, we measure the response functions of the drop properties to the valve actuation [Figs. 3(a) and 3(b)]. For the size control device, we simultaneously measure the drop formation orifice width and the drop size, plotted in Fig. 3(a). The drop size depends nonlinearly on the orifice width because as the valve is actuated, the orifice width is reduced and the local flow velocity is increased; both effects reduce drop size, as expected from the approximation and illustrated by the sharp decrease in drop size at small orifice widths. Nevertheless, for each actuation pressure, monodisperse drops of a controlled size are produced, as shown by the narrow drop size distributions in Fig. 3(c).

By contrast, for the frequency control device, the response function is linear and has a negative slope as a function of the side-channel width; increasing the side-channel width reduces the drop production frequency for fixed actuation amplitude of $2 \mu \mathrm{m}$ and frequency of $30 \mathrm{~Hz}$, as shown in Fig. 3(b). As with the size control device, for each actuation pressure, monodisperse drops are produced with controlled frequency, as shown by the frequency distributions for different actuation pressures in Fig. 3(d).

Valves enable drop properties to be controlled in real time by local adjustment of flow-focus geometry. To illustrate this, we apply an oscillatory actuation to the valves and observe the oscillatory response of the drops. For the size control device, the control and drop signals are plotted as a function of time in Fig. 4(a). The curves are in phase with one another, demonstrating that actuation of the valve modulates, in real time, the size of the drops. To quantify this correlation, we compare the power spectra of the two curves, given in Fig. 3(c). The power spectra track one another up to about $2 \mathrm{~Hz}$ and have a peak at $0.4 \mathrm{~Hz}$, which is the average frequency of the valve actuation and shows that the oscillatory control signal is transferred to the drop train as a modulation in drop size.

For the frequency control device we impose a $10 \mathrm{~Hz}$ periodic signal on the drop train. The control and drop signals are plotted as a function of time for the frequency control device in Fig. 4(b). The curves oscillate in antiphase with one another due to the negative slope of the response function and demonstrating that actuation of the valve modulates, in real time, the frequency of the drops. To quantify this correlation, we again compare the power spectra of the two curves, given in Fig. 4(d). The power spectra track one another up to about $50 \mathrm{~Hz}$ and have a peak at $10 \mathrm{~Hz}$, which is the frequency of the control signal. The maximum periodic frequency we could impose on the drop trains, limited by our pressure regulator, was $1 \mathrm{kHz}$. The valve itself shows no signs of failure up to that frequency.

Valve-based flow focusing allows drop size and frequency to be controlled in real time, over a wide range, and without adjusting flow rate. This control is localized at the flow-focus junction. The localized control increases the modularity of the devices, making them simple to integrate into existing microfluidic chip designs. Valve-based flow focusing should be useful for prototyping new devices or probing large parameter spaces not accessible by changing flow rate alone. It should also be useful for synchronizing coflowing drop trains in droplet microfluidic devices or for encoding information for droplet based logical devices.

This work was supported by Human Frontiers (Grant No. RGP0004/2005-C102), the NSF (Contract Nos. DMR0602684 and DBI-0649865), and the Harvard MRSEC (Contract No. DMR-0213805).

${ }^{1}$ J. Atencia and D. J. Beebe, Nature (London) 437, 648 (2005).

${ }^{2}$ K. Ahn, C. Kerbage, T. P. Hunt, R. M. Westervelt, D. R. Link, and D. A. Weitz, Appl. Phys. Lett. 88, 024104 (2006).

${ }^{3}$ B. H. Weigl, R. L. Bardell, and C. R. Cabrera, Adv. Drug Delivery Rev. 55, 349 (2003)

${ }^{4}$ K. H. Chung, M. M. Crane, and H. Lu, Nat. Methods 5, 637 (2008).

${ }^{5}$ S. Y. Teh, R. Lin, L. Hung, and A. P. Lee, Lab Chip 8, 198 (2008).

${ }^{6}$ A. S. Utada, E. Lorenceau, D. R. Link, P. D. Kaplan, H. A. Stone, and D. A. Weitz, Science 308, 537 (2005).

${ }^{7}$ S. Köster, F. E. Angilè, H. Duan, J. J. Agresti, A. Wintner, C. Schmitz, A. C. Rowat, C. A. Merten, D. Pisignano, A. D. Griffiths, and D. A. Weitz, Lab Chip 8, 1110 (2008).

${ }^{8} \mathrm{~J}$. Xu and D. Attinger, J. Micromech. Microeng. 18, 065020 (2008).

${ }^{9}$ Y. Xia and G. M. Whitesides, Annu. Rev. Mater. Sci. 28, 153 (1998).

${ }^{10}$ D. R. Link, E. Grasland-Mongrain, A. Duri, F. Sarrazin, Z. Cheng, G. Cristobal, M. Marquez, and D. A. Weitz, Angew. Chem. 45, 2556 (2006)

${ }^{11}$ Y.-C. Tan, Y. L. Ho, and A. P. Lee, Microfluid. Nanofluid. 4, 343 (2008).

${ }^{12}$ N.-T. Nguyen, S. Lassemono, and F. A. Chollet, Sens. Actuators B 117 431 (2006).

${ }^{13}$ X. Niu, M. Zhang, S. Peng, W. Wen, and P. Shang, Biomicrofluidics 1 , 044101 (2007).

${ }^{14} \mathrm{~V}$. Barbier, H. Willaime, P. Tabeling, and F. Jousse, Phys. Rev. E 74 046306 (2006).

${ }^{15}$ H. A. Stone, Annu. Rev. Fluid Mech. 37, 387 (2004).

${ }^{16}$ K. P. Johnston, K. L. Harrison,M. J. Clarke, S. M. Howdle, M. P. Heitz, F. V. Bright, C. Carlier, and T. W. Randolph, Science 271, 624 (1996).

${ }^{17}$ A. R. Abate and D. A. Weitz, Appl. Phys. Lett. 92, 243509 (2008).

${ }^{18}$ P. B. Umbanhowar, V. Prasad, and D. A. Weitz, Langmuir 16, 347 (2000).

${ }^{19}$ See EPAPS Document No. E-APPLAB-94-020902 for movies of the devices under operation, as well as AUTOCAD design files for the devices. For more information on EPAPS, see http://www.aip.org/pubservs/epaps.html.

${ }^{20}$ J. Collins and A. P. Lee, Microfluid. Nanofluid. 3, 19 (2006). 